\title{
A TRIBUTE TO MAX PLANCK
}

n Henk Kubbinga - University of Groningen (The Netherlands) - DOI: https://doi.org/10.1051/epn/2018405

Physics seemed complete, in the 1890s. The accomplishments of the kinetic theory of gases were impressive, though some details remained to be solved. The new rays, of all kinds, were just novelties to be embedded in the well-established overall picture. Heat radiation, though, proved disturbing. In its purest form, emitted by a source at constant temperature and, subsequently, refracted by a prism, the spectrum being scanned by a bolometer, it produced curves similar to the statistical distributions current in the kinetic theory. Max Planck was the first to realize the far-reaching consequences. It brought him the Nobel Prize for Physics of 1918.

\section{The coming man; Munich, Kiel}

Max Planck was born in 1858 in Kiel, a city on the northern coast of Germany, as the son of a professor of civil law at the local university. The family moved on to Munich, in 1867, where Planck junior attended a classical gymnasium. His mathematical interests soon prevailed, though in a multifaceted setting, in Germany known as 'Bildung. Classical languages were no problem; philology had charms of its own. Planck equally performed on the piano. At age 16 he enrolled at the University of Munich as a student of physics with mathematics and chemistry as minors. Planck followed courses on general physics by Wilhelm Beetz and more particularly on the mechanical theory of heat, by Philipp von Jolly; the notes he took survived. In 1877-1878 he attended courses of Helmholtz and Kirchhoff at the University of Berlin. A lifelong friendship with Helmholtz was the result. More consequential-that is: from a scientific point of view-were his readings of Rudolf Clausius' chef-d'œuvre Abhandlungen über die mechanische Wärmetheorie (1864-1867). Planck devoted his 'Inaugural dissertation' to the Second Law; entropy kept on fascinating him ever since. Attempts to contact Clausius failed; Clausius wasn't really a man to socialize, it appeared. Undeterred, Planck, in his habilitation lecture (1880), addressed the principles of what he called the 'mechanische Gastheorie'. In 1885 he was-with some help by his father-nominated at the University of Kiel. The courses he came to conduct addressed electricity, optics, and 'mechanische Wärmetheorie'. There were echos of Heinrich Hertz' recent discoveries on the refraction of electromagnetic radiation-waves, apparently-, while there was even chemical thermodynamics in the air. In 1888, then, he was nominated, doubtless through Helmholtz' protection, at the University of Berlin as the successor of Kirchhoff-quite a move for a young scholar ! - , and became the director of the newly established Institute for Theoretical Physics, in the main building of the University at Unter den Linden, not too far from the almost brandnew Physikalisch-Technische Reichsanstalt, at Charlottenburg. It was the year that Clausius passed away, while seeing a new version of his Abhandlungen [..] through

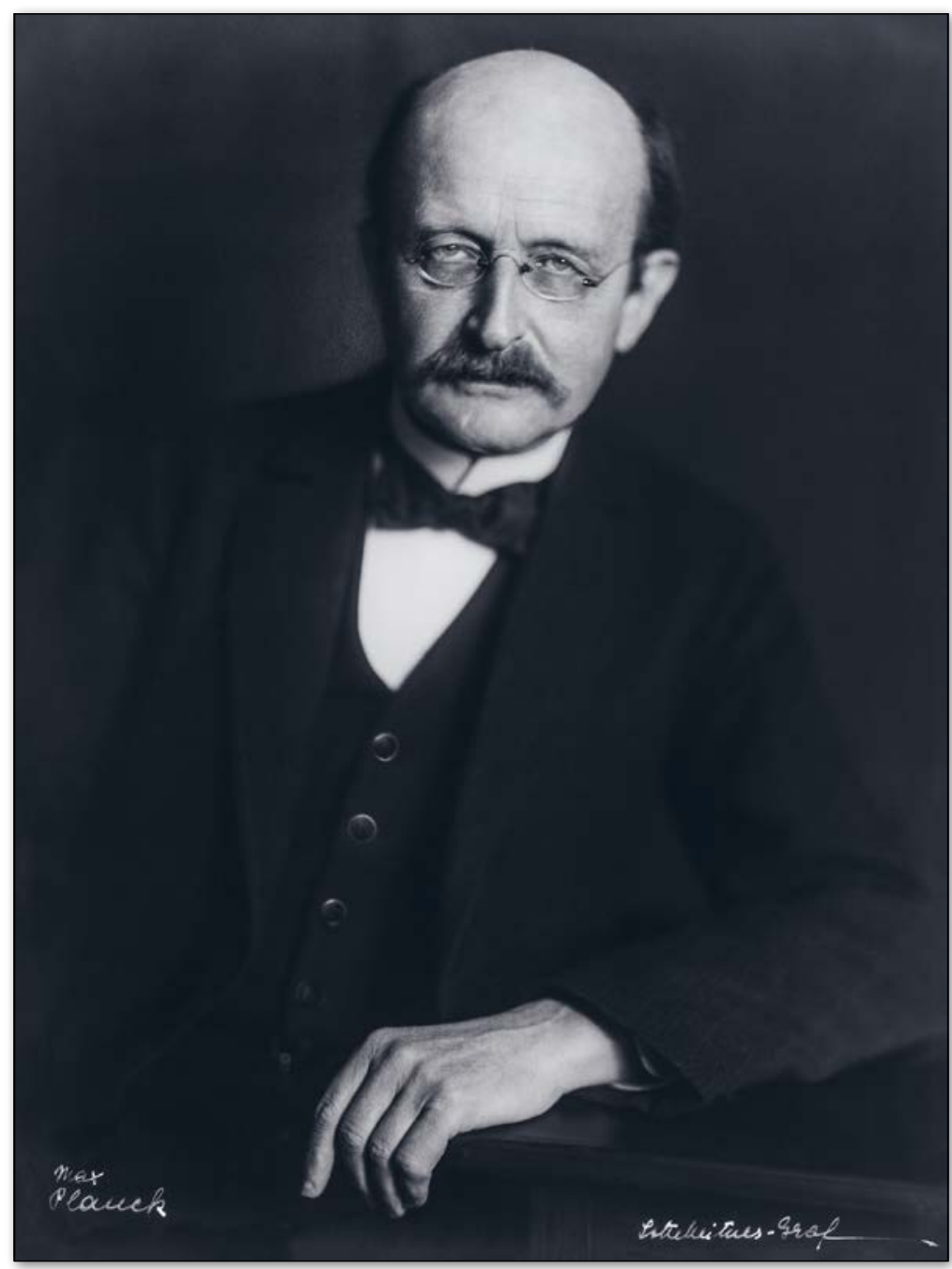

vFIG. 1:

Max Planck (ca. 1930).

Picture made by Lotte Meitner-Graf (courtesy: Lotte Meitner-Graf-Archive; www.LotteMeitnerGraf.com). 
the press; with Carl Pulfrich, a student of Clausius, Planck generously stood in and did what remained to be done, $v i z$., the edition of the third volume, the one devoted to the kinetic theory of gases [1]. Planck and Pulfrich added what Clausius had hinted at, namely Maxwell's statistics. Graphics were still rare. One of the first to include a diagram was Oskar Meyer, in his monograph Die kinetische Theorie der Gase (1877), the title of which would become synonymous with a whole branche of physics (Fig.2). At the time, this was essentially theoretical physics; the proof for its well-foundedness had to wait for 1920 (Otto Stern). As it happened, though, similar curves showed up in thermal radiation phenomena. No wonder, then, that these were associated with gas theory and statistics (Vladimir Michelson; Wien).

\section{Berlin: black body-radiation and statistics}

Kirchhoff's radiating 'schwarze Körper'-only radiation, perfect absorption-, initially a theoretical construct, became of practical importance when light sources had to be standardized at the P.-T.R., Berlin.

VFIG. 2: A generalized curve one of the first in print! - representing the skew distribution function $y=\frac{4}{\sqrt{\pi}} x^{2} e^{-x^{2}}$

for translation velocities in the gaseous state in the spirit of Maxwell. The'most probable velocity' is chosen as the unit (from:

O.E. Meyer, Die kinetische Theorie der Gase, 1877).
The question to be answered: should the city of Berlin be illuminated by gas or electricity? If electricity, what kind of lamp was to be preferred ? Planck, a frequent visitor, got involved when the first high-precision radiation isotherms became available (Fig.3). Wilhelm Wien, of the P.-T.R.-staff, had claimed a relation between the temperature of the radiation source and the wavelength at its curve's maximum, $\lambda_{\max } \cdot T=$ const. Wien also weighted the eventual statistical implications of radiation phenomena, as so strongly suggested by the modalities of the curves, typical skew distributions (1896). It was reserved for Max Planck to find the lacking pieces of the puzzle: the mathematical function behind the curves as an application of permutation theory and, the latter's very essence, the appropriate unit.

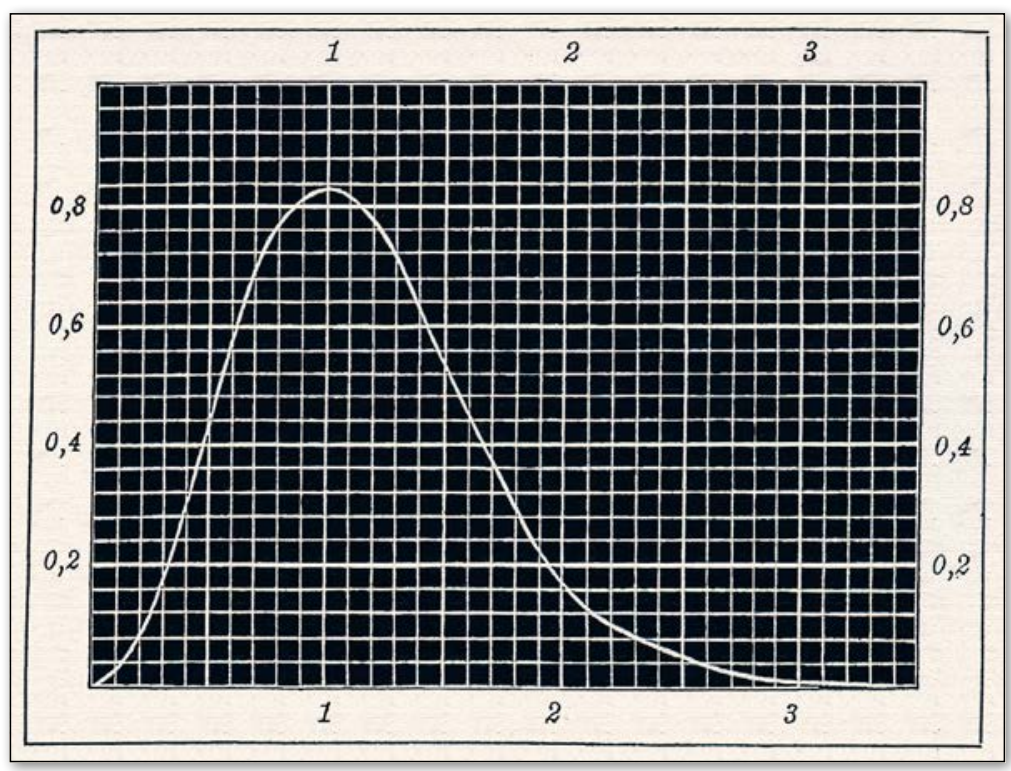

Planck's successive deductions are too well known to be repeated here. Let it suffice, in the present context, to give the adapted function, as published in Planck's epochal 1901 paper, 'Über das Gesetz der Energieverteilung im Normalspektrum' [2]:

$$
\mathrm{U}=\frac{8 \pi c h}{\lambda^{5}} \cdot \frac{1}{e^{c h / k \lambda t-1}}
$$

which defines the energy distribution $U$ as a function of $\lambda$. This inverse fifth-power law, which comes close to one proposed earlier by Wien, features a constant $h$, that needs our particular attention, since Planck's calculation of it - through a transcendent equation-left much to be desired, to say the least. Here we shall present a kind of short-cut.

\section{Gases and radiation}

For someone thoroughly familiar with the kinetic theory of gases, the calculation of the translation velocity of molecules in the gaseous state was straightforward, that is, since 1848, when James Prescott Joule did so for hydrogen gas; his outcome had been $1899 \mathrm{~m} \cdot \mathrm{sec}^{-1}$ for hydrogen gas at $15,6^{\circ} \mathrm{C}$ and $1 \mathrm{~atm}$. Clausius, in 1857 , first derived the general gas law [1]:

$$
p V=1 / 3 n m v^{2}
$$

in which $p$ stands for pressure, $V$ for volume, $n$ for the number of molecules of mass $m$ and velocity $v$. In the light of the Boyle-Gay-Lussac law ( $p V=T \cdot$ const.) Clausius subsequently wrote for the total kinetic energy of his n molecules:

$$
1 / 2 n m v^{2}=3 / 2 T \cdot \text { const. }
$$

where $T$ is the absolute temperature. This expression features only measurable quantities, except for the velocity, $v$. With the product $n m$ as the weight $q$, and $g$ as the gravitational constant Clausius obtained:

$$
v^{2}=\frac{3 g p V}{q}
$$

With the help of the numerical values of the constants involved this expression may be rewritten to become:

$$
v=485 \sqrt{\frac{T}{273 \cdot \rho}}
$$

Hence, the 'mean velocity' of the molecules of a gas at temperature $T$ can be calculated from its density at that temperature. Clausius, then, calculates the 'mean velocity' at $T=273 \mathrm{~K}$ for the molecules of oxygen $\left(461 \mathrm{~m} \cdot \mathrm{sec}^{-1}\right)$, nitrogen $\left(492 \mathrm{~m} \cdot \mathrm{sec}^{-1}\right)$ and hydrogen $\left(1.844 \mathrm{~m} \cdot \mathrm{sec}^{-1}\right)$. All this resurfaced in the third volume overseen by Pulfrich and Planck. It cannot have escaped Planck's inquisitive mind that the reverse calculation was also interesting: when from other sources the 'mean velocity' of a gas' molecules was known, its density $\rho$ 
could be calculated, e.g. for 'theoretical' gases. One of the great experimental achievements of the time, then, was the measurement of the speed of light, $c$, with ever growing precision (Michelson and Morley; 1887 and later). Planck worked in C.G.S. terms and used a value of $3 \cdot 10^{10} \mathrm{~cm} \cdot \mathrm{sec}^{-1}$. So we may expect him to have calculated, a blue Monday, the 'density' of light considered as a 'gas'. In the same spirit we may expect him to have calculated the 'relative weight' of the light 'molecules' involved, that is, with respect to hydrogen, a standard procedure in contemporary chemistry, which he followed as to its thermodynamic aspects. Importantly, the 'molecular' theory of light had a long history: both Newton and Laplace and the latter's followers had been molecularists, so to speak.

\section{A new constant, a calculatio crucis}

The moment that the absolute weight of hydrogen molecules was known, the same quantity could be calculated for all other molecules, real or theoretical, from their velocities. In this connection the number of molecules per $\mathrm{cm}^{3}$ at $273 \mathrm{~K}$, and $1 \mathrm{~atm}$ is crucial. Clausius had guesstimated that number, symbol $N$ at the time, at $3,7 \cdot 10^{19}(1885)$. Given the density of hydrogen as $8,9551 \cdot 10^{-5} \mathrm{~g} \cdot \mathrm{cm}^{-3}(273 \mathrm{~K}, 1 \mathrm{~atm})$ the mass of one hydrogen molecule, then, comes up to $8,9551 \cdot 10^{-5} / 3,7 \cdot 10^{19}=2,42 \cdot 10^{-24} \mathrm{~g}$. Supposing that, at the same $T$, the averaged kinetic energy of gas molecules is the same whatever the gas, we may posit, in C.G.S. units, that the energy of light 'molecules' will be equal to

$$
2,42 \cdot 10^{-24}\left(1844 \cdot 10^{2}\right)^{2}=8,23 \cdot 10^{-14} \mathrm{erg}
$$

On the other hand, that energy must vary directly as the frequency of the light in question. In terms of the radiation curves this is the 'most probable frequency', the one of the curve's maximum. The most reliable data of the moment were those of Lummer and Pringsheim (1899; cf. Fig.3). For a radiating black body of temperature $T$ and $\lambda_{\max }$ as the 'most probable wavelength', Lummer and Pringsheim had established that the product $\lambda_{\max } \cdot T$ of Wien's law was $0,294 \mathrm{~cm} \cdot$ degree. At $T=273^{\circ}$ abs., therefore, $\lambda_{\max }=1,077 \cdot 10^{-3} \mathrm{~cm}$, which corresponds to a frequency $f=3 \cdot 10^{10} / 1,077 \cdot 10^{-3}=2,79 \cdot 10^{13} \mathrm{sec}^{-1}$. We thus need a proportionality constant, Planck's future eponyme, of

$$
8,23 \cdot 10^{-14} / 2,79 \cdot 10^{13}=2,95 \cdot 10^{-27} \mathrm{erg} \cdot \mathrm{sec}^{1} .
$$

Considering that the energy of a light 'molecule' corresponds to $3 k T$, the proportionality constant, later to be called after Boltzmann, appears to be $8,23 \cdot 10^{-14} / 3 \cdot 273=9,75 \cdot 10^{-17} \mathrm{erg} \cdot \mathrm{K}^{-1}$. Since the universal gas constant $\mathrm{R}$ for a quantity of a gas corresponding to one 'g-Molecül'-Planck's term, borrowed from

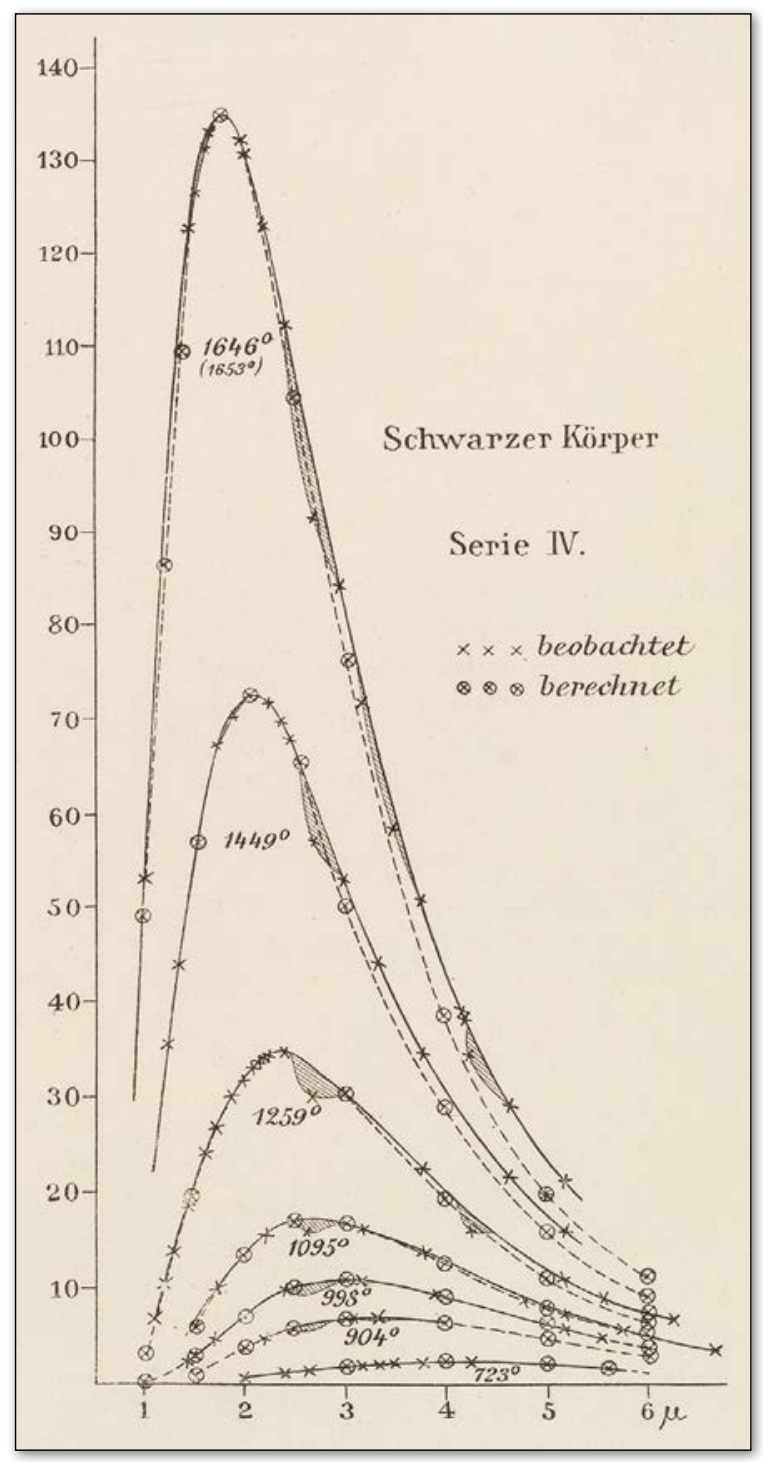

4FIG.3:

Lummer and

Pringsheim 1899 [3]: radiation curves for temperatures from 723 up to $1646^{\circ}$ abs. $\lambda$ is expressed in $\mu$ $\left(10^{-4} \mathrm{~cm}\right)$. Do notice that the curves do not intersect, not unlike the isotherms to be expected in the kinetic theory of gases. thermochemistry-was known to be $8,3 \cdot 10^{7} \mathrm{erg} \cdot \mathrm{K}^{-1}$ we get for the number of molecules in such a 'g-Molecül' $8,3 \cdot 10^{7} / 9,75 \cdot 10^{-17}=8,5 \cdot 10^{23}$. Quite some other constants are now within reach, e.g. the unit charge of electrons and ions.

These numerical values for the searched constants are slightly at variance with those of Planck $\left(h=6,55 \cdot 10^{-27} \mathrm{erg} \cdot \mathrm{sec} ; k=1,346 \cdot 10^{-16} \mathrm{erg} \cdot \mathrm{K}^{-1} ; 6,175 \cdot 10^{23}\right.$ per g-Molecül).

Importantly, the Joule-Clausius-Maxwell line of reasoning taken over by Planck could also be applied to other novelties such as 'electrons'. Joseph John Thomson (1907), for instance, having established that the mass of an electron is $2,6 \cdot 10^{-27}$ gram, calculated in this way their translation velocity in a conductor, taking $v_{\text {hydrogen }}=1700 \mathrm{~m} \cdot \mathrm{sec}^{-1}$ at $273 \mathrm{~K}$ : his outcome was about $10^{5} \mathrm{~m} \cdot \mathrm{sec}^{-1}$. Much later, in 1934, Enrico Fermi similarly calculated the thermal velocity' of neutrons at room temperature $\left(2.600 \mathrm{~m} \cdot \mathrm{sec}^{-1}\right)$.

${ }^{1}$ To be compared with Sommerfeld's values in the range of $2.4 \cdot 10^{-27}$ and $1.4 \cdot 10^{-28} \mathrm{erg} \cdot \mathrm{sec}$ for $\gamma$ - and $\beta$-rays and $8 \cdot 10^{-27} \mathrm{erg} \cdot \mathrm{sec}$ for Röntgen's rays [4]. 


\section{The Nobel Prize}

The Great War had toppled the procedures of the Nobel Foundation. So it came that nominations for three years in a row-1917, 1918 and 1919-were addressed in 1919. Max Planck, then, was awarded the Nobel Prize for Physics of 1918; it was handed over in 1920. He received it, as the Selection Committee summarized its arguments, "in recognition of the service he rendered to the advancement of Physics by his discovery of energy quanta". Among the nominators was Albert Einstein, who, in his letter of October 1918, stressed the importance of Planck's innovations i.a. for Niels Bohr's theory of the relation between spectra and atomic structure. Arnold Sommerfeld referred, on 20 December 1917, to his own work on the new constant for Röntgen's rays, just to show the viability of Planck's approach, but, he too, mentioned Bohr: if Bohr was an obvious candidate for the near future, Sommerfeld argued, he ought to be preceded by Planck. Wilhelm Wien proposed for the same year both Planck and Bohr, in that order; Heinrich Rubens suggested to award the prize to Planck and Bohr together. There were others (Leo Graetz, Carl von Linde and Edgar Meyer), who stressed the importance of Planck's quanta in the study of specific heat at low temperatures, as conducted by Walther Nernst. In all these letters there is, naturally, some window-dressing at stake. The most trustful as to the historical context appears to have been Max Born, in a long letter, dated 20 November 1918. Born indeed recognizes the crucial role of Wien's statistical interpretation along Maxwellian lines (1896), its failure for longer wavelengths, Planck's new formula (based on Boltzmann's use of permutation-theory), and the indispensable energie-elements.

\section{Acknowledgment}

I am indebted to Karl Grandin (Royal Swedish Academy of Sciences, Stockholm; Chair EPS-HoP Group) for kindly allowing me to consult the letters nominating Planck for the the 1918 and 1919 Nobel Prize for Physics. For documentary photography I am obliged to Tony Barrett (Lotte Meitner-Graf Archive, Cambridge) and Dirk Fennema (University of Groningen).

\section{About the author}

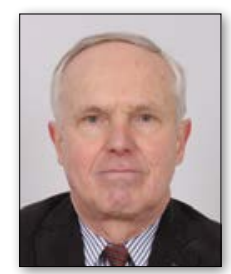

Henk Kubbinga is a historian of science at the University of Groningen and member of the EPS-HoP-Group. Actually he is finishing the fifth and last volume of The collected papers of Frits Zernike (1888-1966).

\section{References}

For background reading see J. Mehra and H. Rechenberg's multivolume The historical development of quantum theory (New York etc.: Springer-Verlag, 1982-) and D. Hoffmann, Max Planck. Die Entstehung der modernen Physik, Munich: C.H. Beck, 2008. Both works skip the details of Planck's calculation. For the broad context see my The molecularization of the world picture (Groningen: Groningen University Press, 2009; a German edition is forthcoming).

[1] R. Clausius, Die kinetische Theorie der Gase, C. Pulfrich and M. Planck (eds.), Brunswick: F. Vieweg, 1889-1891.

[2] M. Planck, Ann.d.Phys. 4, 553 (1901).

[3] H. Lummer and E. Pringsheim, Verhand.Deut.Phys.Ges. 1, 215 (1899).

[4] A. Sommerfeld, Sitz. [.] der K. Bayer. Ak. d. Wiss. München 1911, 1-60.

\section{ПคП口ПЕТค 2019}

